\title{
L'humanisation du droit international:la personne humaine en tant que sujet du droit des gens ${ }^{1}$
}

\section{Antônio Augusto Cançado Trindade ${ }^{2}$}

\section{Résumé}

L'individu est sujet jure suo de droit international, comme soutenu au fil des siècles par la doctrine juridique plus lucide, dès les écrits des célèbres "pères fondateurs" de notre discipline. L'importance considérable attribuée à la personne humaine dans le cadre du droit des gens par les célèbres "pères fondateurs"de la discipline ne devrait pas être oubliée à notre époque. La soumission postérieure de l'individu à la "volonté" de l'État n'a jamais parue convaincante, et a vite été ouvertement remise en question par la doctrine juridique plus lucide. À notre époque, l'individu est reconnu - et de manière très claire dans la doctrine jusinternationaliste grecque - comme sujet à la fois de droit interne et de droit international. La subjectivité internationale de l'être humain est apparue avec vigueur dans la science juridique du $\mathrm{XX}^{\mathrm{e}}$ siècle, comme réaction de la conscience juridique universelle contre les atrocités successives commises contre le genre humain. L'accès direct de l'individu à la juridiction internationale est donc entièrement justifié pour défendre ses droits, même contre son propre État.

\footnotetext{
${ }^{1}$ Discurso proferido pelo Autor na sessão solene de outorga do título de Doutor Honoris Causa da Universidade Panteion de Atenas, realizada no salão-nobre da referida Universidade, em Atenas, Grécia, em 01 de julho de 2014.

${ }^{2}$ Professor Emérito de Direito Internacional da Universidade de Brasília (UnB); Juiz da Corte Internacional de Justiça (Haia); Ex-Presidente da Corte Interamericana de Direitos Humanos; Presidente Honorário do Instituto Brasileiro de Direitos Humanos; Presidente da Sociedade Latinoamericana de Direito Internacional; Membro Titular do Institut de Droit International e do Curatorium da Academia de Direito Internacional da Haia.
} 
La consolidation de la personnalité juridique internationale des individus renforce pari passu la responsabilité en droit international pour des abus perpétrés contre les êtres humains.En cedébut de $\mathrm{XXI}^{\mathrm{e}}$ siècle, cette conquête très importante peut être appréciée dans le cadre du processus historique en cours de l'humanisation du droit international.

\section{Abstract}

The individual is subject jure suo of international law, as sustained along the centuries by the most lucid legal doctrine, as from the writings of the renowned "founding fathers" of our discipline. The considerable importance attributed to the human person in the framework of the law of nations by the famous "founding fathers" of the discipline should not be forgotten in our epoch. The subsequent submission of the individual to the "will" of the State has never appeared convincing, and was promptly challenged by the most lucid legal doctrine. In our epoch, the individual is recognized - and quite clearly in Greek jusinternationalist doctrine - as subject of domestic as well as international law. The international subjectivity of the human being has emerged with vigour in the legal science of the XXth century, as a reaction of the universal juridical conscience against the successive atrocities committed against the human kind. The direct access of the individual to the international jurisdiction is thus entirely justified so as to defend his rights, even against his own State. The consolidation of the international legal personality of individuals enhances pari passu responsibility in international law for the abuses perpetrated against human beings. At this beginning of the XXIth century, this very important advance can be appreciated in the framework of the historical process in course of the humanization of international law. 


\section{Resúmen}

El individuo es sujeto jure suo de derecho internacional, como sustentado a lo largo de los siglos por la doctrina jurídica más lúcida, desde los escritos de los célèbres "padres fundadores" de nuestra disciplina. La importancia considerable atribuída a la persona humana en el marco del derecho de gentes por los famosos "padres fundadores"de la disciplina no debería ser olvidada en nuestra época. La sujeción posterior del individuo a la "voluntad" del Estado jamás pareció convincente, y fue prontamente cuestionada por la doctrina juridica más lúcida. En nuestra época, el individuo es reconocido - y de modo marcante en la doctrina jusinternacionalista griega - como sujeto del derecho tanto interno como internacional. La subjectividad internacional del ser humano emergió con vigor en la ciencia jurídica del siglo XX, como reacción de la consciencia jurídica universal contra las atrocidades succesivas conmetidas contra el género humano. El acceso directo del individuo a la juridicción internacional es, pues, enteramente justificado para defender sus derechos, aún contra su proprio Estado. La consolidación de la personalidad jurídica internacional de los individuos refuerza pari passu la responsabilidad en derecho internacional por los abusos perpetrados contra los seres humanos. En este inicio del siglo XXI, este avance muy importante puede ser apreciado en el marco del proceso histórico en curso de la humanización del derecho international.

\section{Resumo}

O indivíduo é sujeito jure suo do direito internacional, como sustentado ao longo dos séculos pela doutrina jurídica mais lúcida, desde os escritos dos célèbres "pais fundadores" de nossa disciplina. A importancia considerável 
atribuída à pessoa humana no âmbito do direito das gentes pelos famosos "pais fundadores"da disciplina não deveria ser esquecida em nossa época. A submissão posterior do indivíduo à "vontade" do Estado jamais pareceu convincente, e foi prontamente questionada pela doutrina juridíca mais lúcida. Em nossa época, o indivíduo é reconhecido - e de modo marcante na doutrina jusinternacionalista grega - como sujeito do direito tanto interno como internacional. La subjetividade internacional do ser humano emergiu com vigor na ciencia jurídica do século $\mathrm{XX}$, como reação da consciencia jurídica universal contra as atrocidades sucessivas cometidas contra o gênero humano. $\mathrm{O}$ acesso direto do indivíduo à jurisdição internacional é, pois, inteiramente justificado para defender seus derechos, mesmo contra seu próprio Estado. A consolidação da personalidade jurídica internacional dos indivíduos reforça pari passu a responsabilidade no direito internacional pelos abusos perpetrados contra os seres humanos. Neste início do século XXI, este avanço muito importante pode ser apreciado no âmbito do processo histórico em curso da humanização do direito international.

\section{La Présence et la Participation de la Personne Humaine dans l'Ordre Juridique International en tant que Sujet du Droit des Gens}

J'aimerais commencer par exprimer ma gratitude aux autorités de l'Université Panteion d'Athènes, pour avoir pris l'initiative de cette démarche académique de ce soir, le $1^{\text {er }}$ juillet 2014. C'est un grand honneur pour moi d'être nommé Docteur Honoris Causa, et cela me donne l'occasion d'échanger personnellement, ici à 1'Université Panteion $\mathrm{d}$ 'Athènes, avec mes chers collègues et amis de l'École grecque contemporaine de droit international, avec lesquels j'ai partagé au cours 
des dernières années des moments mémorables dans le milieu académique, à la fois en Europe et en Amérique Latine. Je remercie, en particulier, M. Le Recteur, le Professeur Grigoris Tsaltas, de l'attention de son addresse d'ouverture, et M. Le Vice-Recteur, le Professeur Stelios Perrakis, de la gentillesse de sa Laudatio, très significative pour moi. J'aimerais ajouter qu'il y a déjà longtemps, j'ai appris à apprécier le précieux héritage de l'époque hellénistique, qui a été marquée par une rare prospérité de l'apprentissage et des arts, qui a eu des répercussions dans différentes latitudes sur les siècles qui l'ont suivie.

La Grèce ancienne a été témoin de l'apparition de la grammaire et de la critique de textes (en tant que nouvelle discipline), de la littérature elle-même (poésie, tragédies et comédies); elle a cultivé la philosophie et la sculpture; elle a vu les théâtres se multiplier; elle a influencé la naissance ultérieure du droit (à l'époque romaine), avec un sens profond de la justice. De façon significative, la profonde influence du stoïcisme dans la pensée hellénistique a ouvert la voie à l'humanisme. Nous avons des raisons d'être reconnaissants, en Europe et en Amérique Latine, pour l'héritage grec que représente la pensée humaniste.

C'est dans le cadre de cette pensée humaniste que je propose de traiter mon sujet aujourd'hui, lors de la cérémonie organisée dans cette Université Panteion d'Athènes. Il s'agit du sujet suivant: 'L 'humanisation du Droit international: La personne humaine en tant que sujet du droit des gens / The Humanization of International Law: The Human Person as Subject of the Law of Nations". Pour apprécier les développements dans le droit des gens contemporain, il est nécessaire de commencer par se tourner, même brièvement, vers les origines de notre discipline, et donc vers les origines du jus gentium tel qu'il est venu à être compris, englobant des Etats, des peuples et des individus. 


\section{La personne humaine dans la pensée jusnaturaliste du droit des gens}

L'importance considérable attribuée à la personne humaine dans le cadre du droit des gens par les célèbres"pères fondateurs"de la discipline ne devrait pas être oubliée à notre époque. Au cours du XVI ${ }^{\mathrm{e}}$ siècle, la conception de Francisco de Vitoria (auteur des célèbres Relecciones Teológicas, 1538-1539) s'est développée, et selon elle, le droit des gens régule une communauté internationale (totus orbis) constituée d'êtres humains organisés socialement en Etats et conformant l'humanité; la réparation des violations des droits (de l'homme) reflète une nécessité internationale satisfaite par le droit des gens, avec les mêmes principes de justice s'appliquant à la fois aux États et aux individus et peuples qui les forment $^{3}$. Au XVII ${ }^{\mathrm{e}}$ siècle, dans la vision avancée par Francisco Suárez (auteur du traité De Legibus ac Deo Legislatore, 1612), le droit des nations révèle l'unité et l'universalité de l'humanité et régule les Etats dans leurs relations en tant que membres de la société universelle.

Peu de temps après, au XVII ${ }^{\mathrm{e}}$ siècle, la conception élaborée par Hugo Grotius (De Jure Belli ac Pacis, 1625), supporte l'idée que la societas gentium comprend l'ensemble de l'humanité et la communauté internationale ne peut pas prétendre se baser sur la voluntas de chaque État individuel; les êtres humains - occupant une position centrale dans les relations internationales - ont des droits vis-à-vis de l'État souverain qui ne peuvent pas forcer l'obéissance de leur citoyens de manière absolue (l'impératif du bien commun), car la fameuse "raison d'État" a ses limites et ne peut pas se détourner du Droit. Dans cette ligne de pensée, encore au XVII ${ }^{\mathrm{e}}$ siècle, Samuel Pufendorf (De Jure Naturae et Gentium, 1672) soutient

\footnotetext{
3. À son tour, Alberico Gentili (auteur de De Jure Belli, 1598) soutenait, à la fin du XVI siècle, que le Droit gouverne les relations entre les membres de la societas gentium universelle.
} 
aussi l'idée de l'assujettissement du législateur à la raison alors que, au XVIII $^{\mathrm{e}}$ siècle, Christian Wolff (Jus Gentium Methodo Scientifica Pertractatum, 1749) pense que comme les individus devraient - dans leur association à l'Etat - promouvoir le bien commun, l'Etat, à son tour, a le devoir corrélatif de rechercher sa perfection. ${ }^{4}$

La personnification ultérieure de l'État tout puissant, inspirée surtout de la philosophie du droit de Hegel, a eu une influence néfaste sur l'évolution du droit international à la fin du $\mathrm{XIX}^{\mathrm{e}}$ siècle et dans les premières décennies du $\mathrm{XX}^{\mathrm{e}}$ siècle. Cette tendance doctrinale a résisté du mieux qu'elle pouvait à l'idéal de l'émancipation de l'être humain du contrôle absolu de l'Etat et à la reconnaissance de l'individu comme sujet de Droit International. Mais la soumission de l'individu à la "volonté" de l'État n'a jamais parue convaincante, et a vite été ouvertement remise en question par la doctrine plus lucide. L'idée d'une souveraineté absolue de l'Etat, - qui a conduit à l'irresponsabilité et à la présumée omnipotence de l'Etat, n'empêchant pas les atrocités successives commises par lui (ou en son nom) contre les êtres humains, - est apparue avec le passage du temps comme entièrement non fondée.

Dans son étude sur La morale internationale (1944), publié pendant la IIe. guerre mondiale, N. Politis attirait l'attention, avec des références à des leçons des tragédies d'Eschyle et $\mathrm{d}^{\prime}$ Euripide $^{5}$, sur le nécessaire équilibre - toujours avec tension - entre la légalité et la justice. La pensée humaine, aux XVIIIe et XIXe siècles, se montrait déjà consciente de cet équilibre nécessaire; cette conviction a graduellement

\footnotetext{
${ }^{4}$ A.A. Cançado Trindade, “A Consolidação da Personalidade e da Capacidade Jurídicas do Indivíduo como Sujeito do Direito Internacional", 16 Anuario del Instituto Hispano-LusoAmericano de Derecho Internacional - Madrid (2003) pp. 240-247; A.A. Cançado Trindade, "Vers la consolidation de la capacité juridique internationale des pétitionnaires dans le système interaméricain des droits de la personne", in 14 Revue québécoise de Droit international (2001) n. 2, pp. 207-239.

5 . N. Politis, La morale internationale, New York, Brentano's, 1944, pp. 100, 102 et 157.
} 
commencé à pénétrer dans la conscience humaine ${ }^{6}$. N. Politis a averti, avec perspicacité, qu'“'à] la différence des profits de l'injustice et de l'illégalité, qui, s'ils peuvent être rapides, ne sont pas assurés de durer, ceux de la justice et de la légalité, sans doute plus lents, sont certainement plus durables"7. Au cours de tout le XXe siècle, la pensée humaniste a resisté aux tyrannies, et a reconnu la nécessité impérieuse des valeurs, beaucoup plus importantes que la volonté ${ }^{8}$.

L'État - il est reconnu de nos jours - est responsable de tous ses actes (à la fois jure gestionisetjure imperii), ainsi que de toutes ses omissions ${ }^{9}$. En cas de violation des droits de l'homme, l'accès directde l'individu à la juridiction internationale est donc totalement justifié pour défendre ces droits, même contre son propre État ${ }^{10}$. À partir des travaux ayant résulté en une résolution prise par l'Institut de Droit International en 1929, S. Séfériadès a donné un cours à l'Académie de Droit International de La Haye en 1935, dans lequel il a soutenu l'accès direct des individus à des juridictions internationales, pour améliorer "la justice de ce monde". Il s'agissait d’une vraie nécessité; à son avis,

"[l]a protection diplomatique s'efface ainsi complètement lorsque la justice apparaît. Devant elle, ce n'est plus l'État protecteur qui se présente, mais, directement, le particulier interéssé lui-même: c'est celui-ci qui parle"11.

6. Ibid., p. 158, et cf. p. 164.

7. Ibid., pp. 161-162.

${ }^{8}$. Cf. R.P. Sertillanges, Le problème du mal - l'Histoire, Paris, Aubier, 1948, pp. 395397.

9 . Ibid., pp. 247-259.

${ }^{10} \mathrm{~S}$. Glaser, "Les droits de l'homme à la lumière du droit international positif", in Mélanges offerts à H. Rolin - Problèmes de droit des gens, Paris, Pédone, 1964, pp. 117-118, et cf. pp. 105-106 et 114-116.

11. S. Séfériadès, "Le problème de l'accès des particuliers à des juridictions internationales", 51 Recueil des Cours de l'Académie de Droit International de La Haye (1935) p. 31, et cf. pp. 48, 51, 94, 106 et 112. Le thème de la condition de l'individu dans l'ordre juridique international est démeuré présent dans les divers écrits de S. Séfériadès, dans le contexte des problèmes de son époque, cf. S. Séfériadès, "L'échange des populations", 24Recueil des Cours de l'Académie de Droit International de La Haye 
À notre époque, l'individu est reconnu comme sujet à la fois de droit interne et de droit international. En fait, il est toujours resté en contact, directement ou indirectement, avec l'ordre juridique international ${ }^{12}$. Pendant la période de l'entre-deux-guerres, les expériences des minorités et des systèmes des mandats de la Société des Nations, par exemple, en témoignent. Ils ont été suivis, à cet égard, du système de tutelle de l'ère de l'Organisation des Nations Unies, en parallèle au développement par cette dernière de multiples mécanismes - conventionnels et extra-conventionnels - de protection internationale des droits de l'homme. Ces premières expériences $\mathrm{au} \mathrm{XX}^{\mathrm{e}}$ siècle étaient importantes pour les développements ultérieurs en matière de sauvegarde internationale des droits de la personne humaine.

L'évolution considérable dans les dernières dizaines d'années non seulement du Droit International des Droits de l'Homme mais similairement du Droit International Humanitaire, a contribué de manière décisive à la réaffirmation du contact constant de l'individu avec l'ordre juridique international. Le Droit International Humanitaire considère également les personnes protégées non seulement comme des simples objets de la réglementation qu'elles établissent mais comme vrais sujets de Droit International ${ }^{13}$. C'est ce qui découle clairement du fait que les quatre Conventions de Genève interdisent clairement aux Etats parties de déroger par accords spéciaux - aux règles que ces Conventions énoncent, et en particulier de restreindre les droits des personnes protégées qu'elles

(1928) pp. 311-433; S. Séfériadès, "Principes généraux du droit international de la paix", 34 Recueil des Cours de l'Académie de Droit International de La Haye (1930) pp. 310$313,315,317,320$ et 322-323.

${ }^{12}$ Cf. A.A. Cançado Trindade, "The Historical Recovery of the Human Person as Subject of the Law of Nations", 1 Cambridge Journal of International and Comparative Law (2012) pp. 8-59.

13. C'est ce qui résulte, e.g., de la position des quatre Conventions de Genève sur le Droit International Humanitaire de 1949, érigée à partir des droits des personnes protégées (e.g., Convention III, articles 14 et 78; Convention IV, article 27). 
prévoient ${ }^{14}$. En effet, l'impact des normes du Droit International des Droits de l'Homme a eu depuis longtemps des répercussions sur le corpus juris et la mise en œuvre du Droit International Humanitaire. Par conséquent, le Droit International Humanitaire s'est progressivement libéré d'une vision obsolète purement interétatique, mettant de plus en plus l'accent - au regard des principes d'humanité - sur les personnes protégées et sur la responsabilité de la violation de leurs droits, de conformité avec la ligne de pensée jusnaturaliste du jus gentium et de la civitas maxima gentium $^{15}$.

Les tentatives du passé de refuser aux individus la condition de sujets de Droit International du fait que l'on ne leur reconnaissait pas certaines compétences que les Etats avaient (comme par exemple celle de l'élaboration de traités) sont définitivement dépourvues de sens ${ }^{16}$. Cette tendance doctrinale, tentant d'insister sur une définition tellement rigide de la subjectivité internationale, conditionnant cette dernière à la formation des normes internationales et à leur respect, ne se tient tout simplement pas, pas même au niveau du droit interne dans lequel il n'est pas requis que chaque individu - il n'a d'ailleurs jamais été requis - participe à la création et la mise en œuvre de normes juridiques pour être sujet (titulaire) de droits et être lié par les devoirs émanant de ces normes.

En plus d'être non viable, cette conception semble contaminée par un dogmatisme idéologique inquiétant qui a eu comme conséquence principale

\footnotetext{
${ }^{14}$. Conventions de Genève I, II et III, Article 6; et Convention de Genève IV, Article 7. En fait, dès le passage du XIXe au XXe siècle, les premières Conventions sur le Droit International Humanitaire exprimaient des inquiétudes quant au sort des êtres humains dans les conflits armés, reconnaissant ainsi l'individu comme bénéficiaire direct des obligations conventionnelles internationales.
}

15. Cf. M. Huber, La pensée et l'action de la Croix-Rouge, Genève, CICR, 1954, pp. 26, 247, 270, 286, 291-293 et 304.

16. Au niveau interne, les individus ne participent pas non plus en totalité, directement ou indirectement, au processus législatif, et ils ne cessent pas pour autant d'être sujets de droit. 
d'aliéner l'individu de l'ordre juridique international. Il est surprenant,- sinon étonnant, - en plus d'être regrettable, de voir cette conception répétée mécaniquement et ad nauseam par une tendance doctrinale qui essaie apparemment de faire croire que la fonction intermédiaire de l'Etat entre les individus et l'ordre juridique international serait quelque chose d'inévitable et de permanent. Rien ne pourrait être plus faux. Dans la période historique brève dans laquelle cette conception étatique prévalait, au regard du positivisme juridique, des atrocités successives ont été commises contre l'être humain à une échelle sans précédent.

Il en résulte assez clairement aujourd'hui qu'il n'y a rien d'intrinsèque au Droit International qui empêche les acteurs non étatiques, ou qui leur rend impossible, de bénéficier d'une personnalité juridique internationale. Personne n'ose en bonne conscience nier que les individus sont effectivement titulaires des droits et porteurs des obligations qui émanent directement du Droit International avec lequel il se trouvent ainsi en contactdirect. Et il est parfaitement possible de conceptualiser - même avec plus de précision - comme sujet de droit international toute personne ou entité titulaire de droits et d'obligations qui émanent directement des normes de Droit International. C'est le cas des individus, qui ont ainsi renforcé ce contact direct - sans intermédiaires - avec l'ordre juridique international. Le mouvement international en faveur des droits de l'homme, lancé par la Déclaration Universelle des Droits de l'Homme de 1948, a fini par ne plus autoriser les fausses analogies susmentionnées et par surmonter les distinctions traditionnelles (par exemple, sur la base de la nationalité) : les sujets de droit sont tous des êtres humains en tant que membres de la societas gentium universelle ${ }^{17}$.

\footnotetext{
${ }^{17}$ R. Cassin, "L'homme, sujet de droit international et la protection des droits de l'homme dans la société universelle", dansLa technique et les principes du Droit public - Études en l'honneur de G. Scelle, vol. I, Paris, LGDJ, 1950, pp. 81-82.
} 
De plus, de nos jours, les individus et les organisations non gouvernementales (ONGs) jouent un rôle de plus en plus important dans la formation même de l'opinio juris communis ${ }^{18}$. Au cours de ces derniers décennies, les individus et les ONGs ont efficacement participé aux travaux préparatoires de certains traités internationaux, et les ont influencés ${ }^{19}$, et ont ensuite participé à la surveillance de leur mise-en-œuvre. La performance croissante, au niveau international, des ONGs et des autres entités de la société civile a eu un impact inévitable sur la théorie des sujets de Droit International, contribuant à rendre les individus non seulement bénéficiaires directs (sans intermédiaires) des normes internationales, mais sujets réels de droit international, et contribuant aussi à mettre un terme à la dimension anachronique purement interétatique de celui-ci; de plus, leurs activités ont contribué à la prévalence des valeurs communes supérieures dans le cadre du droit international. Les individus, les ONGs et les autres entités de la société civile finissent ainsi par agir dans le processus de formation et de mise-enapplication des normes internationales.

\section{La consécration de la personne humaine comme sujet du droit des gens}

\footnotetext{
${ }^{18}$. Les ONGs ont gagné une visibilité considérable au cours du récent cycle de Conférences Mondiales de 1'O.N.U. (1992-2001) par leur présence dans les Conférences mêmes ou par leur articulation dans leurs propres fora en parallèle avec ces Conférences. Dans ces dernières années, elles ont eu le droit de présenter régulièrement leurs amici curiae devant les tribunaux internationaux tels que les Cours Interaméricaines et Européenne des Droits de l'Homme, et les Tribunaux Pénaux Internationaux ad hoc pour l'ex-Yougoslavie et pour le Rwanda.

19. E.g., la Convention de l'O.N.U. de 1984 contre la Torture et son Protocole Facultatif de 2002, la Convention de l'O.N.U. de 1989 des Droits de l'Enfant, le Protocole de Madrid de 1991 (au Traité sur l'Antarctique de 1959) sur la Protection de l'Environnement en Antarctique, la Convention d'Ottawa de 1997 sur l'Interdiction des Mines Antipersonnel et sur leur Elimination, Le Statut de Rome de 1998 relatif à la Cour Pénale Internationale, et la Convention de l'UNESCO de 2005 sur la Protection et la Promotion de la Diversité des Expressions Culturelles.
} 
En somme, le processus même de formation et de mise-en-œuvre des normes de droit international a cessé d'être un monopole des Etats. De plus, au-delà de la présence et de la participation de l'individu dans l'ordre juridique international, à la reconnaissance de ses droits en tant que sujet de droit international doit correspondre la capacité procédurale de les défendre au niveau international. Depuis déjà plusieurs années, je soutiens la nécessité du legitimatio ad causampleine des individus en droit international $^{20}$. C'est au moyen de la consolidation de la capacité procédurale internationale des individus que la protection internationale des droits de l'homme devient réelle et effective ${ }^{21}$. Même si, au travers des circonstances de la vie, certains individus (par exemple les enfants, les malades mentaux, les personnes âgées, parmi d'autres) ne peuvent pas exercer entièrement leur capacité (par exemple en droit civil), cela ne veut pas dire qu'ils cessent d'être titulaires de droits opposables même aux États. Quelles que soient les circonstances, l'individu est sujet jure suo de droit international, comme soutenu au fil des siècles par la doctrine plus lucide, dès les écrits des célèbres "pères fondateurs" de notre discipline. Les droits de l'homme sont conçus comme inhérents à chaque être humain, indépendamment des circonstances.

Bien que le scénario international contemporain soit entièrement distinct de celui de l'époque des célèbres“pères fondateurs" du droit international (personne ne peut le nier) qui ont avancé une civitas maxima

20. A.A. Cançado Trindade, El Acceso Directo del Individuo a los Tribunales Internacionales de Derechos Humanos, Bilbao, Universidad de Deusto, 2001, pp. 17-96; A.A. Cançado Trindade, "The Procedural Capacity of the Individual as Subject of International Human Rights Law: Recent Developments”, dans K. Vasak Amicorum Liber - Les droits de l'homme à l'aube du XXIe siècle, Bruxelles, Bruylant, 1999, pp. 521-544.

21. Cf. A.A. Cançado Trindade, El Acceso Directo del Individuo a los Tribunales Internacionales..., op. cit. supra n. (18), pp. 17-96; A.A. Cançado Trindade, "Vers la consolidation de la capacité juridique internationale des pétitionnaires dans le système interaméricain des droits de la personne", 14 Revue québécoise de Droit international (2001) pp. 207-239. 
régie par le droit des gens, il y a une aspiration humaine récurrente, transmise de génération en génération au cours des siècles, menant à la construction d'un ordre juridique international applicable à la fois aux États (et organisations internationales) et aux individus, conformément à certains standards universels de justice. Cela explique l'importance, dans ce nouveau corpus juris de protection, que la personnalité juridique internationale de l'individu a assumé, étant à la fois sujet de droit interne et de droit international.

L'individu, étant sujet de Droit International de plein droit, pouvait certainement être distingué de son propre État, et toute faute commise à son encontre était une violation du jus gentium classique, un droit minimal universel. L'ensemble du nouveau corpus juris du Droit International des Droits de l'Homme a été construit sur la base des impératifs de protection et des intérêts supérieurs de l'être humain, sans tenir compte de son lien de nationalité ou de son statut politique, ou de quelque autre situation ou circonstance. Cela explique l'importance, dans ce nouveau droit de protection, de la personnalité juridique de l'individu comme sujet à la fois de droit interne et de droit international. La mise en ouvre et l'expansion du Droit International des Droits de l'Homme a ensuite eu des répercussions, non surprenantes, avec un impact sensible sur les tendances du Droit International Public contemporain ${ }^{22}$.

En fait, déjà dans les premiers décennies du $\mathrm{XX}^{\mathrm{e}}$ siècle, l'on reconnaissait les insuffisances et dérangements occasionnés par la protection des individus par l'intermédiaire de leurs États de nationalité respectifs, c'est-

${ }^{22}$.Cf. A.A. Cançado Trindade, Tratado de Direito Internacional dos Direitos Humanos, vol. I, 2nd. ed., Porto Alegre/Brazil, S.A. Fabris Ed., 2003, pp. 33-50, et vol. II, 1999, pp. 23-194 ; A.A. Cançado Trindade, O Direito Internacional em um Mundo em Transformação, Rio de Janeiro, Ed. Renovar, 2002, pp. 1048-1109 ; A.A. Cançado Trindade, El Derecho Internacional de los Derechos Humanos en el Siglo XXI, Santiago, Editorial Jurídica de Chile, 2001, pp. 15-58 et 375-427. 
à-dire, par l'exercice d'une protection diplomatique discrétionnaire qui rendait les Etats “plaignants" à un moment “juges et parties”. Par conséquent, on a commencé à surmonter ces insuffisances et dérangements, à nourrir l'idée de l'accès direct des individus à la juridiction internationale dans certaines conditions, pour défendre leurs droits même contre les États, - un thème qui a fini par être en fait envisagé par l'Institut de Droit International dans ses sessions de 1927 et 1929. En Europe ${ }^{23}$, aussi qu'en Amérique Latine $^{24}$, même avant l'adoption des Déclarations Américaine et Universelle des Droits de l'Homme (de mai et décembre1948, respectivement), des manifestations doctrinales se sont exprimées, et ont évolué en faveur de la personnalité juridique internationale des individus. Comme réaction de la conscience juridique universelle, on a conçu la responsabilité internationale comme comprenant, à la fois, la protection des droits de l'homme et la punition des criminels de guerre (formant un ensemble).

Ce développement a annoncé l'émancipation de l'individu de la tutelle de son propre État, et sa condition de sujet de droit international; puisque l'individu est "sujet de devoirs" au niveau du droit international, l'on ne peut ignorer sa personnalité juridique internationale reconnue aussi en fait par le droit international coutumier même ${ }^{25}$.Bien avant

${ }^{23}$.N. Politis, Les nouvelles tendances du Droit international, Paris, Libr. Hachette, 1927, ch. II, pp. 55-92; J. Spiropoulos, L'individu en Droit international, Paris, LGDJ, 1928, pp. 366;A.N. Mandelstam, Les droits internationaux de l'homme, Paris, Éds. Internationales, 1931, pp. 95-96 et 138, et cf. p. 103; G. Scelle, Précis de Droit des Gens - Principes et systématique, part I, Paris, Libr. Rec. Sirey, 1932 (CNRS reprint, 1984), pp. 42-44 et 48; H. Lauterpacht, International Law and Human Rights, London, Stevens, 1950, pp. 51, 61 et 6970; M. Bourquin, "L'humanisation du droit des gens", dans La technique et les principes du Droit public ..., op. cit. supra n. (15), vol. I, pp. 21-54.

${ }^{24}$. A. Álvarez, La Reconstrucción del Derecho de Gentes - El Nuevo Orden y la Renovación Social, Santiago de Chile, Ed. Nascimento, 1944, pp. 46-47, 81, 91, 457-463 et 499-500; H. Accioly, Tratado de Direito Internacional Público, vol. I, 1e. éd., Rio de Janeiro, Imprensa Nacional, 1933, pp. 71-75.

${ }^{25}$ C.Th. Eustathiades, "Les sujets du Droit international et la responsabilité internationale Nouvelles tendances", 84 Recueil des Cours de l'Académie de Droit International de La 
ce développement si significatif, il faut garder à l'esprit que, déjà en 1927, dans son essai Les nouvelles tendances du Droit international, Nicolas Politis avait averti que 1'État "n'est pas une fin en soi"; 1'État est soumis au Droit, et le droit (international et interne)

"a toujours la même fin: il vise partout l'homme, et rien que l'homme. Cela est tellement évident, qu'il serait inutile d'y insister si les brumes de la souveraineté n'avaient pas obscurci les vérités les plus élémentaires" ${ }^{\text {"26. }}$.

Les milieux sociaux (les sociétés internationale et interne), - il a ajouté, “n'existent que pour assurer à l'homme la possibilité de vivre et de se développer" 27 .

Dans son livre L'individu en droit international, publié une année plus tard, en 1928, Jean Spiropoulos avait affirmé que l'ordre juridique international contient des droits aussi bien que de devoirs "visant directement les individus", qui deviennent ainsi sujets de droit international; celui-ci les rend directement titulaires de droits et obligations $^{28}$. J. Spiropoulos avait critiqué durement la philosophie du droit de Hegel, qui a vu dans l'État "un idéal suprême, un but en soi, une puissance qui n'est soumise qu'à sa propre volonté" ${ }^{29}$, et qui a résisté "de toutes ses forces"à cette émancipation de l'individu de la tutelle de l'État ${ }^{30}$. Par contre, il y a eu une prise de conscience de la nécessité de protéger l'individu "même contre son propre État". Alors, le droit international a commencé à s'occuper de l'émancipation de l'individu de la toute-

Haye (1953) pp. 402, 412-413, 424-427, 547, 586-589, 601, 608 et 610-612; P.

Guggenheim, "Les principes de Droit international public", 80 Recueil des Cours de

l'Académie de Droit International de La Haye (1952) pp. 116-118.

26. N.Politis, Les nouvelles tendances du Droit international, Paris, Libr. Hachette, 1927, pp. 76-78.

27 . Ibid., pp. 78-79.

28. J. Spiropoulos, L'individu en droit international, Paris, LGDJ, 1928, pp. 31-32.

29 . Ibid., p. 55.

${ }^{30}$. Ibid., p. 33, et cf. pp. 19 et 66. 
puissance et de la tutelle de 1'État ${ }^{31}$. Cette émancipation de l'individu a commencé à se manifester par la "participation directe de l'individu à la vie juridique internationale" 32 .

Pour J. Spiropoulos, l'État n'est pas un "fin en soi”, n'est pas une "toute puissance personnifiée" au sens de la philosophie de Hegel; 1'État est "une simple 'communauté d'administration' d'intérêts humains". Et 1'État, - contrairement à ce que disait Hegel, - "est soumis au droit international" 33 , et doit satisfaire les besoins des individus qui le composent $^{34}$. On peut ajouter ici encore une autre contribution sur le même thème, le premier ouvrage de Georges Ténékidès ( $L$ índividu dans l'ordre juridique international, 1933) ${ }^{35}$. La condition de l'individu comme sujet du droit international est alors devenue une importante contribution de la pensée jusinternationaliste grecque de la période d'entre-guerres, dans sa ligne d'orientation essentiellement humaniste. Dès cette époque et jusqu'à présent, la doctrine juridique grecque n'a jamais abandonné ce thème, et continue à le cultiver de nos jours.

$\mathrm{Au}$ milieu du $\mathrm{XX}^{\mathrm{e}}$ siècle, dans les premières années de la mise en œuvre de la Convention Européenne relative aux Droits de l'Homme, il y avait du soutient pour la vision selon laquelle les individus étaient devenus titulaires de droits, puisqu'un processus d'émancipation des individus de la tutelle "exclusive" del'État avait déjà commencé en droit international. Dans la doctrine juridique de ce temps, la reconnaissance de l'expansion de la protection internationale de la personne humaine est devenue évidente.La victoire de cette pensée sur le positivisme juridique était rassurante, puisque

\footnotetext{
31. Ibid., p. 44.

32 . Ibid., p. 49.

33. Ibid., pp. 62-64.

${ }^{34}$. Ibid., p. 66.

35. G. Ténékidès, L índividu dans l'ordre juridique international, Paris, Pédone, 1933, pp. 3-263.
} 
l'individu, titulaire de droits et devoirs internationaux, n'était plus à la merci de son État, car l'humanité s'est tourné vers le droit naturel à la recherche du bien-être de la personne humaine, "alors que le droit international positif actuel tend à sa destruction" 36 .

En fait, des études successives d'instruments de protection internationale en sont venues à mettre l'accent précisément sur l'importance historique de la reconnaissance de la personnalité juridique internationale des individus comme partie plaignante devant des organes internationaux. Dans mon propre coursdélivré à l'Académie de Droit International de la Haye en 1987, j'ai soutenu que l'expansion continue du Droit International était aussi reflétée dans les multiples mécanismes contemporains de protection internationale des droits de l'homme, dont l'opération ne pourrait être dissociée des nouvelles valeurs reconnues par la communauté internationale ${ }^{37}$. Enfin, les individus ont réussi à exercer leurs droits émanant directement du droit international (le droit des gens); et j'ai ajouté que, historiquement,

"les mêmes principes de justice s'appliquant à la fois aux Etats et aux individus ou peuples qui les forment.(...) Il y a une reconnaissance croissante et généralisée que les droits de l'homme, au lieu de découler de l'Etat (...), sont tous inhérents à la personne humaine en laquelle ils trouvent leur point de convergence ultime. (...) Le non respect des droits de l'homme engendre la responsabilité internationale des Etats du traitement de la personne humaine" 38 .

La subjectivité internationale de l'être humain (qu'il soit un enfant, une personne âgée, une personne handicapée, une personne apatride, ou

36. B.V.A. Röling, International Law in an Expanded World,Amsterdam, Djambatan, 1960, p. 2.

37. A.A. Cançado Trindade, "Co-existence and Co-ordination of Mechanisms of International Protection of Human Rights (At Global and Regional Levels", 202 Recueil des Cours de l'Académie de Droit International de La Haye(1987) pp. 32-33.

${ }^{38}$. Ibid., pp. 411-412. 
quelque autre personne que ce soit) est apparue avec vigueur dans la science juridique du $\mathrm{XX}^{\mathrm{e}}$ siècle, comme réaction de la conscience juridique universelle contre les atrocités successives commises contre le genre humain. Un témoignage éloquent de l'érosion de la dimension purement interétatique de l'ordre juridique international est trouvé dans l'Avis Consultatif pionnier (n. 16) de la CIADH sur le Droit à l'Information sur l'Assistance Consulaire dans le Cadre des Garanties duProcès Équitable (du 01.10.1999) ${ }^{39}$, qui a servi d'orientation à d'autres tribunaux internationaux et a inspiré l'évolution in statu nascendi de la jurisprudence internationale sur la question; la CIADH a reconnu la cristallisation d'un vrai droit subjectif individuel à l'information sur l'assistance consulaire ${ }^{40}$ dont chaque être humain dépourvu de sa liberté dans un autre pays est titulaire $^{41}$; de plus, elle s'est détachée de la perspective traditionnelle purement interétatique sur la question, apportant son appui aux divers individus victimes de la pauvreté etde la discrimination, et dépourvus de liberté à l'étranger.

L'Avis Consultatif suivant (n. 17) de la CIADH, sur la Condition Juridique et les Droits del'Enfant (du 28.08.2002), rentre dans le même type d'affirmation de l'émancipation juridique de l'être humain en mettant

\footnotetext{
39. CIADH, Avis Consultatif OC-16/99, Série A, n. 16, pp. 3-123, paras. 1-141, et points 18.
}

${ }^{40}$ Indiqué dans l'article 36 de la Convention de Vienne de 1963 sur les Relations Consulaires et lié aux garanties de jugement en bonne et due forme conformément à l'article 8 de la Convention Américaine sur les Droits de l'Homme.

${ }^{41}$ Dans cet Avis, la CIADI a clairement indiqué que les droits mentionnés dans l'article 36(1) de la Convention de Vienne de 1963 sur les Relations Consulaires "ont comme caractéristique le fait que leur titulaire est l'individu. En effet, cette disposition est explicite en déclarant que les droits à l'information consulaire et la notification sont 'accordés' à la personne intéressée. A cet égard, l'article 36 est une exception notable à la nature essentiellement étatique des droits et obligations mentionnés ailleurs dans la Convention de Vienne sur les Relations Consulaires ; comme interprété par cette Cour dans l'Avis Consultatif présent, cela représente une avancée notable pour ce qui est des conceptions traditionnelles du Droit International sur le sujet" (paragraphe 82, accent ajouté). 
l'accent sur la consolidation de la personnalité juridique de l'enfant en tant que vrai sujet de droit et non comme simple objet de protection, et quelle que soit l'étendue de sa capacité juridique à exercer ses droits par lui-même (capacité d'exercer). À cet égard, la Convention de l'O.N.U. sur les Droits de l'Enfant (1989) reconnaît les droits subjectifs de l'enfant en tant que sujet de droit, et reconnaît également qu'étant donné sa vulnérabilité ou sa condition existentielle, l'enfant a besoin d'attention et de représentation juridique spéciales tout en restant titulaire de droits. On a reconnu la nécessitéde fournir une protection aux êtres humains qui la composent, en particulier à ceux qui se trouvent dans une situation de vulnérabilité particulière.

Selon la doctrine juridique de la seconde moitié du $\mathrm{XX}^{\mathrm{e}}$ siècle, il n'est pas passé inaperçu que les individus, en plus d'être titulaires de droits au niveau international, ont aussi des devoirs qui leur sont attribués par le droit international même. Et, - ce qui est plus important, - la violation grave de ces devoirs, reflétée dans les crimes contre l'humanité, engage la responsabilité pénale individuelle internationale, indépendamment de ce que prévoit le droit interne sur la question.Des développements contemporains en droit pénal international ont en fait une incidence directe sur la cristallisation de la responsabilité pénale individuelle internationale (l'individu comme sujet, à la fois actif et passif, de droit international, titulaire de droits ainsi que porteur de devoirs émanant directement du droit des nations (droit des gens), ainsi que sur le principe de juridiction universelle.

\section{La consolidation de la personnalité juridique internationale de la personne humaine et l'humanisation du droit des gens}


La consolidation de la personnalité juridique internationale des individus, comme sujets actifs aussi bien que passifs de droit international, renforce la responsabilité en droit international pour des abus perpétrés contre les êtres humains. Par conséquent, les individus sont aussi porteurs de devoirs en droit international, et cela reflète la consolidation de leur personnalité juridique internationale. Les développements en matière de personnalité juridique internationale et de responsabilité internationale vont de pair, et toute cette évolution témoigne de la formation de l'opinio juris communis au point que la gravité de certaines violations de droits fondamentaux de la personne humaine affecte directement les valeurs fondamentales de la communauté internationale dans son ensemble.

Au bout du compte, tout Droit existe pour l'être humain et le droit des gens ne fait pas exception, garantissant à l'individu ses droits et le respect de sa personnalité. Le respect de la personnalité juridique de l'individu au niveau international est instrumentalisé par le droit international de pétition individuelle. Les droits de l'homme s'affirment contre toute forme de domination ou de pouvoir arbitraire ${ }^{42}$. Sans le droit de pétition individuelle et l'accès conséquent à la justice au niveau international, les droits établis dans les traités relatifs aux droits de l'homme seraient réduits à un peu plus qu'une lettre morte.Comme j'avais souligné dans mon Opinion Concurrente dans l'affaire de Castillo Petruzzi et Autres (Exceptions Préliminaires, Arrêt du 04.09.1998) devant la CIADH,

"Le droit de pétition individuelle abrite en fait le dernier espoir de ceux qui n'ont pas trouvé la justice au niveau international. Je ne m'empêcherai pas

${ }^{42}$ A.A. Cançado Trindade, "The Future of the International Protection of Human Rights", in B. Boutros-Ghali Amicorum Discipulorumque Liber - Paix, Développement, Démocratie, vol. II, Bruxelles, Bruylant, 1998, pp. 961-986. - Sur le besoin de surmonter les défis et les obstacles actuels à la prévalence des droits de l'homme, cf. A.A. Cançado Trindade, "L'interdépendance de tous les droits de l'homme et leur mise-en-œuvre: obstacles et enjeux", 158 Revue internationale des sciences sociales - Paris/UNESCO (1998) pp. 571-582. 
d'ajouter - me permettant la métaphore - que le droit de pétition individuelle est sans aucun doute l'étoile la plus lumineuse de l'univers des droits de l'homme",43.

L'être humain émerge enfin, même dans les conditions les plus défavorables, comme sujet ultime de droit interne et international. Comme j'ai trouvé bon de résumer dans mon Opinion Concurrente dans l'Avis Consultatif susmentionné de la CIADH sur la Condition Juridique et les Droits de l'Enfant (2002),

\begin{abstract}
“chaque être humain est doté de personnalité juridique qui impose des limites au pouvoir de l'Etat. La capacité juridique varie en vertu de la condition juridique de chacun pour entreprendre certains actes. Cependant, bien qu'une telle capacité d'exercice varie, tous les individus sont dotés de personnalité juridique. Les droits de l'homme renforcent l'attribut universel de la personne humaine, étant donné qu'à tous les êtres humains correspond similairement la personnalité juridique et la protection du Droit, indépendamment de sa condition existentielle ou juridique"(paragraphe 34).
\end{abstract}

Pour ce qui est des droits de l'homme des individus appartenant aux groupes ou collectivités humaines, je me permets de me référerà l'Avis Consultatif n. 18 de la CIADH, déjà célèbre, sur la Condition Juridique et Droits des Migrants Sans Papiers (du 17.19.2003).La Cour Interaméricaine a mis l'accent sur le fait que le statut migratoire ne peut pas servir de justification pour les priver de la jouissance et de l'exercice de leurs droits fondamentaux, y compris le droit du travail. La Cour a averti que les Étatsne peuvent pas subordonner le respect du principe fondamental d'égalité devant la loi et la non-discrimination aux objectifs de leurs politiques migratoires ou autres, ni le conditionner à celles-ci ${ }^{44}$.

43. CIADH, affaire Castillo Petruzzi et Autres contre Pérou (Exceptions Préliminaires), Arrêt du 04.09.1998, Opinion Concordante du Juge A.A. Cançado Trindade, p. 62, para. 35.

44. Dans mon Opinion Concurrente je soutenais l'idée que ce principe fondamental appartenait au domaine du jus cogens et j'ai mis l'accent sur l'importance des obligations 
La subjectivité juridique internationale de l'être humain, telle que prévue par les célèbres"pères fondateurs" du droit international (le droit des gens) est à présent une réalité. En ce début de $\mathrm{XXI}^{\mathrm{e}}$ siècle, cette conquête très importante peut être appréciée dans le cadre du processus historique de l'humanisation du droit international, - auquel c'est un privilège de pouvoir contribuer, - qui, toujours attentif aux valeurs fondamentales, finit par s'occuper plus directement de la réalisation des objectifs communs supérieurs. Dans le cadre du Droit International des Droits de l'Homme, dans les systèmes européen et interaméricain de protection - dotés de tribunaux internationaux qui fonctionnent depuis longtemps - parallèlement à la personnalité juridique, la capacité juridique internationale (le locus standi in judicio) des individus est aussi reconnue.

Cela est un développement logique puisqu'il ne semble pas raisonnable de concevoir des droits au niveau international sans la capacitéjuridique correspondante pour les défendre; les individus sont en fait la vraie partie plaignante dans le contentieux international relatif aux droits de l'homme. Sur la base du droit de pétition individuelle, le mécanisme juridique d'émancipation de l'être humain est érigé par rapport à son propre État pour la protection de ses droits dans le cadre du Droit International des Droits de l'Homme, - une émancipation qui constitue de nos jours une vraie révolution juridique qui arrive enfin à donner un contenu éthique aux normes à la fois du droit public interne et du droit internationalpublic.

La reconnaissance de l'accès direct des individus à la justice internationale révèle, en ce début de $\mathrm{XXI}^{\mathrm{e}}$ siècle, la nouvelle primauté de la raison de l'humanité par rapport à la raison d'Etat, ce qui inspire le

erga omnes (englobant aussi les relations interindividuelles) vis-à-vis des droits des immigrants sans papiers. 
processus historique d'humanisation du droit international ${ }^{45}$. Les sujets de droit international ont, déjà depuis longtemps, cessé d'être réduits à des entités territoriales $^{46}$. Il semble assez clair aujourd'hui qu'il n'y a rien d'intrinsèque au droit international qui empêcherait, ou rendrait impossible, aux "acteurs" non étatiques d'être dotés de personnalité et de compétence juridique internationale.

L'expansion de la personnalité juridique internationale, englobant de nos jours celle des individus comme sujets actifs et passifs de droit international, va pari passu avec l'expansion de la responsabilité en droit international.Cela contribue en final à la preéminence du droit (rule of law), à la réalisation de la justice aussi au niveau du droit international, répondant ainsi aux aspirations de longue date de l'humanité. En réaction aux atrocités successives qui, au cours du $\mathrm{XX}^{\mathrm{e}}$ siècle, ont fait des millions et des millions de victimes humaines à une échelle que l'histoire de l'humanité n'avait pas connue jusque là, la conscience juridique universelle - comme source matérielle ultime de tout Droit ${ }^{47}$, - a rendu à l'être humain sa condition de sujet de droit, à la fois du droit interne et du droit international, et sa condition de destinataire final de toutes les normes juridiques, d'origine nationale ainsi qu'internationale. Le droit

45. A.A. Cançado Trindade, A Humanização do Direito Internacional, Belo Horizonte/Brésil, Edit. Del Rey, 2006, pp. 3-409;A.A. Cançado Trindade, Évolution du Droit international au droit des gens - L'accès des particuliers à la justice internationale: le regard d'un juge, Paris, Pédone, 2008, pp. 140-144.

46. Il y a plus d'un siècle, comme reconnu dans le célèbre Avis Consultatif de la Cour Internationale de Justice (CIJ) sur les Réparations desDommages (1949), l'apparition $d$ 'organisations internationales avait mis un terme au monopole des Etats en termes de personnalité et capacité juridiques internationales, avec toutes les conséquences juridiques qui en ont découlé, cf., pour une étude générale sur la question, A.A. Cançado Trindade, Direito das Organizações Internacionais, 5e. éd., Belo Horizonte/Brazil, Edit. Del Rey, 2012, pp. 9-853.

47. Cf. A.A. Cançado Trindade, Le Droit international pour la personne humaine, Paris, Pédone, 2012, pp. 91-112. 
international s'est libéré des chaines de l'étatisme, en avançant la conception du nouveau jus gentium ${ }^{48}$.

\section{The Centrality of the Human Person in the Humanization of Contemporary International Law}

Je me permets de continuer mon allocution en anglais. International law, as we know it today, does not go back only to the XIXth century, - as a doctrinal trend en vogue nowadays tries in vain to make one believe, - but goes much further back in time, keeping in mind its conceptual framework, and the endeavours, along centuries, to fulfill the aspirations of the whole of human kind. Regrettably, the vision and thinking of the so-called "founding fathers" of International Law (notably the writings of the Spanish theologians and of $\mathrm{H}$. Grotius), which conceived it as conforming a truly universal ${ }^{49}$ system, came to be replaced by the emergence of legal positivism, which personified the State, endowing it with its own "will", reducing the rights of human beings to those which were "conceded" by the State. The subsequent personification of the all-powerful State, inspired mainly in the philosophy of law of Hegel, had a harmful influence in the evolution (or rather involution) of International Law by the end of the XIXth century and in the first decades of the XXth century.

48. A.A. Cançado Trindade, International Law for Humankind - Towards a New Jus Gentium, $2^{\text {nd }}$. rev. ed., Leiden/The Hague,Nijhoff/The Hague Academy of International Law, 2013, pp. 1-726.

${ }^{49}$. C.W. Jenks, The Common Law of Mankind, London, Stevens, 1958, pp. 66-69; and cf. also R.-J. Dupuy, La communauté internationale entre le mythe et l'histoire, Paris, Economica/UNESCO, 1986, pp. 164-165. 
Theconsent or will of the States became, according to voluntarist positivism, the predominant criterion in international law, denying jus standi to individuals, to the human beings. This rendered difficult the understanding of the international community, weakening International Law itself, reducing it to a strictly inter-State law, no longer above but between sovereign States. This doctrinal trend resisted as much as it could to the ideal of the emancipation of the human being from the absolute control of the State, and to the recognition of the individual as subject of international law.

Against the reactionary dogmas of legal positivism stood, among others, Jean Spiropoulos, in a thoughtful monograph titled L'individu en Droit international, published in Paris in $1928^{50}$ : contrary to what ensued from the Hegelian doctrine, - he pondered, - the State is not a supreme ideal subjected only to its own will, is not an end in itself, but rather "a means of the realization of the aspirations and vital necessities of the individuals", it thus being necessary to protect the human person against the harm to her rights by her own State ${ }^{51}$.

Positivists, ascribing a far too great importance to the method of observation, disclosed, in contrast, their incapacity to discern guidelines of analysis and guiding general principles. At normative level, positivism appeared subservient to the established legal order, and validated the abuses perpetrated in its name. But already in the mid-XXth century, the more lucid doctrine of the law of nations moved definitively away from the hegelian and neo-Hegelian outlook of the State as the final repository of the freedom and responsibility of the individuals who composed it, and which in it integrated themselves entirely. After all, States, created by the human beings

\footnotetext{
${ }^{50}$ J. Spiropoulos, L'individu en Droit international, Paris, LGDJ, 1928, pp. 66 and 33, and cf. p. 19.

51. Ibid., p. 55; an evolution to this effect, - he added, - would bring us closer to the ideal of civitas maxima.
} 
themselves, and composed by them, exist for them, for the realization of their common good.

\section{The Atribution of Duties to the Individual Directly by International Law}

To the legal doctrine of the second half of the XXth century it did not pass unnoticed that individuals, besides being titulaires of rights at international level, also have duties which are attributed to them by international law itself. And, - what is more significant, - the grave violation of those duties, reflected in the crimes against humanity, engages the international individual penal responsibility, independently from what provides the domestic law on the matter. Contemporary developments in international criminal law have, in fact, a direct incidence in the crystallization of both of the international individual penal responsibility (the individual subject, both active and passive, of international law, titulaire of rights as well as bearer of duties emanated directly from the law of nations (droit des gens), as well as the the principle of universal jurisdiction.

The consolidation of the international legal personality of individuals, as active as well as passive subjects of international law, enhances accountability in international law for abuses perpetrated against human beings. Thus, individuals are also bearers of duties under international law, and this reflects the consolidation of their international legal personality. Developments in international legal personality and international accountability go hand in hand, and this whole evolution bears witness of the formation of the opinio juris communis to the effect that the gravity of certain violation of fundamental rights of the human person affects directly basic values of the international community as a whole. 


\section{Subjective Right, Human Rights and the New Dimension of the International Juridical Titularity of the Human Person}

The international juridical titularity of the human person, as socalled "founding fathers" of international law (the law of nations, the droit des gens) foresaw it, is nowadays a reality. Furthermore, the (active) international subjectivity of the individuals responds to a true necessity of their legitimatio ad causam (cf. supra), to vindicate their rights, emanated directly from International Law. In the ambit of the International Law of Human Rights, in the regional (European, inter-American and African) systems of protection - endowed with international tribunals in operation one recognizes today, parallel to the legal personality, also the internacional procedural capacity (locus standi in judicio) of the individuals.

This is a logical development, as it would not appear reasonable to conceive rights at international level without the corresponding procedural capacity to vindicate them; theindividual applicants are effectively the true complaining party in the international contentieux of human rights. Upon the right of individual petition is erected the juridical mechanism of the emancipation of the human person vis-à-vis the State itself for the protection of her rights in the ambit of the International Law of Human Rights, - an emancipation which constitutes, in our days, a true juridical revolution, which comes at last to give an ethical content to the norms of both public domestic law and international law.

On the basis of this remarkable development lies the principle of respect for the dignity of the human person, irrespective of her existential condition. By virtue of this principle, every human being, independently of his situation and of the circumstances in which he finds himself, has the right 
to dignity. The whole remarkable development of the jusinternationalist doctrine in this respect, along the XXth century, finds its roots, - and it could not be otherwise, - in some reflections of the past, in the juridical as well as philosophical thinking, - as exemplified, inter alia (to refer to one which goes far back in time), by the Kantian conception of the human person as an end in itself. This is ineluctable, as it reflects the process of maturing and refinement of the human spirit itself, which renders possible the advances in the human condition itself.

In effect, one cannot dissociate the recognition of the international legal personality of the individual (supra) from the dignity itself of the human person. In effect, it is the human person, essentially endowed with dignity, the one who articulates, expresses and introduces the "Sollen" of the values in the world of the reality wherein she lives, and it is only her who is capable of doing so, as bearer of such ethical values. The legal personality, for its part, is manifested as a juridical category in the universe of Law, as theunitary expression of the aptitude of the human person to betitulaire of rights and bearer of duties at the level of regulated behaviour and human relations ${ }^{52}$.

It may be recalled, in the present context, that the conception of individual subjectiveright has already a wide historical projection, originated in particular in the jusnaturalist thinking in the XVIIth and XVIIIth centuries, and systematized in the legal doctrine throughout the XIXth century. However, in the XIXth century and beginning of the XXth century, that conception remained situated in the ambit of domestic public law, emanated from the public power. Even so, the crystallization of the concept of individual subjective right, and its systematization, marked a step forward towards a better comprehension of the individual as titulaire of rights. And they rendered it possible to attain, with the emergence of human rights at international level, the gradual overcoming of positive law.

${ }^{52}$. Cf., in this sense, e.g., L. Recaséns Siches, Introducción al Estudio del Derecho, 12th. ed., Mexico, Ed. Porrúa, 1997, pp. 150-151, 153, 156 and 159. 
By the mid-XXth century, the impossibility became clear of the evolution do Law itself without the individual subjective right, expression of a true "human right" 53 .As I deemed it fit to sustain in my Concurring Opinion in the historical Advisory Opinion n. 16 of the IACtHR on the Right to Information on Consular Assistance in the Ambit of the Guarantees of the Due Process of Law (of 01.10.1999), we nowadays witness

"the process of humanization of international law, which today encompasses also this aspect of consular relations. In the confluence of these latter with human rights, the individual subjective right to information on consular assistance, of which are titulaires all human beings who are in the need to exercise it, has crystallized: such individual right, situated into the conceptual universe of human rights, is nowadays supported by conventional international law as well as by customary international law" (par. 35) ${ }^{54}$.

The emergence of universal human rights, as from the proclamation of the Universal Declaration of 1948, came to widen considerably the horizon of contemporary legal doctrine, disclosing the insufficiencies of the traditional conceptualization of the subjective right. The pressing needs of protection of the human being much fostered this development. Universal human rights, superior and anterior to the State and to any form of sociopolitical organization, and inherent to the human being, asserted themselves as opposable to public power itself. Human rights freed the conception of subjective right (supra) from the chains of legal positivism. If, on the one hand, the juridical category of the international legal personality of the human being contributed to instrumentalize the vindication of the rights of the human person, emanated from international law, - on the other hand the

53. J. Dabin, El Derecho Subjetivo, Madrid, Ed. Rev. de Derecho Privado, 1955, p. 64.

54. On the impact of this Advisory Opinion n. 16 (of 1999) of the IACtHR on contemporary international case-law and practice, cf. A.A. Cançado Trindade, "The Humanization of Consular Law: The Impact of Advisory Opinion n. 16 (1999) of the InterAmerican Court of Human Rights on International Case-Law and Practice", in 6 Chinese Journal of International Law (2007) n. 1, p. 1-16. 
corpus juris of universal human rights ascribed to the legal personality of the individual a far wider dimension, no longer conditioned to the law emanated from the public State power.

Also in the International Court of Justice (ICJ), I have recently had the occasion, in my Separate Opinion appended to the Advisory Opinion of the ICJ on theRevision of a Judgment of the ILO Administrative Tribunal upon a Complaint Filed against IFAD(01.02.2012), to dwell upon the outdated dogmatism of the PCIJ and ICJ Statutes, in so far as the international legal capacity of individuals is concerned. As I have recalled in that Separate Opinion (pars.70-75), the question of the procedural capacity of the individuals before the ICJ, and its predecessor the Permanent Court of International Justice (PCIJ), was effectively considered on the occasion of the original drafting, by the Advisory Committee of Jurists appointed by the old League of Nations, of the Statute of the PCIJ, in $1920^{55}$. Of the ten members of the aforementioned Committee of Jurists, only two - Loder and De La Pradelle - pronounced themselves in favour of enabling the individuals to appear as parties before The Hague Court (jus standi) in contentious cases against (foreign) States. The majority of the Committee, however, was firmly opposed to this proposition ${ }^{56}$.

The position which prevailed in 1920 - which has been surprisingly and regrettably maintained in Article 34(1) of the Statute of the ICJ (formerly

\footnotetext{
55. A.A. Cançado Trindade, El Acceso Directo del Individuo..., op. cit. supra n. (18), p. 31, and cf. pp. 32-35.

56. Four members (Ricci-Busatti, Baron Descamps, Raul Fernandes and Lord Phillimore) objected that the individuals were not subjects of international law (and could not, thus, in their view, be parties before the Court) and that only the States were juridical persons in the international order, - in what they were followed by the other members. Cf. account inJ. Spiropoulos, L'individu en Droit international, Paris, LGDJ, 1928, pp. 50-51; N. Politis, op. cit. infra n. (44), pp. 84-87; M.St. Korowicz, "The Problem of the International Personality of Individuals", 50 American Journal of International Law (1956) p. 543; S. Séfériadès, "Le problème de l'accès des particuliers à des juridictions internationales",op. cit. supra n. (9), pp. 46-47.
} 
the PCIJ) to date - was promptly and strongly criticized in the more lucid doctrine of the epoch (already in the twenties - cf. supra). Thus, - to recall the Greek international legal thinking, - in his aforementioned thoughtful monograph Les nouvelles tendances du Droit international (1927), Nicolas Politis, after remarking that all Law aims ultimately at human beings (who compose States), proceeded in the defence of the granting to individuals of the direct recourse to international instances in order to vindicate their "legitimate interests", as that would fulfill "a true necessity of international life" $"$.

Another criticism to the solution adopted in the matter by the Statute of the PCIJ (Article 34(1)) was formulated by J. Spiropoulos, also in the twenties. Already in 1928, he had anticipated that the emancipation of the individual from the State was a "question of time" and that the individual should be able to defend himself and his rights at the international level ${ }^{58}$. There was - he added - no impediment for conventional International Law to secure to individuals a direct action at international level (there having even been precedents in this sense in the inter-war period); if this did not occur and one would limit oneself to judicial actions at domestic law level, not seldom the State would become "judge and party" at the same time, what would be an incongruity.

To J. Spiropoulos, the international legal order can address itself directly to individuals (as exemplified by the peace treaties of the inter-war period), thereby erecting them into the condition of subjects of international law, to the extent that a direct relationship is established between the individual and the international legal order, which renders him "directly titulaire of rights or of obligations"; thus, one cannot fail to admit the

57. N. Politis, Les nouvelles tendances du Droit international, Paris, Libr. Hachette, 1927, pp. 69, 76-78, 82-83 and 89-90, and cf. pp. 92 and 61.

58 . J. Spiropoulos, op. cit. supra n. (48), p. 44, and cf. pp. 49 and 64-65. 
international legal personality of the individual ${ }^{59}$. Without the granting to individuals of direct means of action at international level, his rights will continue "without sufficient protection"; only with such direct action before an international instance, - he added, - an effective protection of human rights will be achieved, in conformity with the "spirit" of the new international order.

In the mid-thirties, the same position, as already pointed out, was taken by $S$. Séfériadès ${ }^{60}$.In the same line of thinking are the subsequent writings of Georges Ténékidès, as from his first book ( $L$ índividu dans l'ordre juridique international,1933) ${ }^{61}$; he likewise remained always attentive to the central position of individuals in the law of nations, having related it to the law of international organizations ${ }^{62}$, and having pursued an inter-disciplinary approach ${ }^{63}$. Almost five decades later, he lectured at the Hague Academy of International Law on the action of the United Nations against all forms of racial discrimination ${ }^{64}$.

The option made by the draftsmen of the Statute of the old PCIJ in 1920, stratified with the passing of time in the Statute of the ICJ up to the present time, is even more open to criticism if we consider that, already in the first half of the XXth century, there were experiments of International

\footnotetext{
${ }^{59}$. Ibid., pp. 50-51, 25, 31-33 and 40-41.

60. Cf. S. Séfériadès, "Le problème de l'accès des particuliers à des juridictions internationales", op. cit. supra n. (9), pp. 31-32, 51-52, 94 et 106.

${ }^{61}$. Cf. G. Ténékidès, L'individu dans l'ordre juridique international, Paris, Pédone, 1933, pp. 3-263.

62. G. Ténékidès, "Régimes internes et organisation internationale", 110 Recueil des Cours de l'Académie de Droit International de La Haye (1963) pp. 405-408.
}

63. Ibid., pp. 398-415; and cf. G. Ténékidès, "L'uniformité des régimes politiques au sein des Ligues et Confédérations grecques à l'époque classique", inVölkerrecht und Rechtliches Weltbild (eds. K. Zemanek et alii), Vienna, Springer-Verlag, 1960, pp. 263271.

64. G. Ténékidès, "L'action des Nations Unies contre la discrimination raciale", 168 Recueil des Cours de l'Académie de Droit International de La Haye (1980) pp. 285-467. 
Law which in effect granted international procedural status to individuals ${ }^{65}$.This evolution intensified and generalized in the era of the United Nations, with the adoption of the system of individual petitions under some universal human rights treaties of our times, in addition to human rights conventions at regional level, which established international human rights tribunals (the European and Inter-American Courts of Human Rights, followed, more recently, by the African Court of Human and Peoples' Rights). Thereunder the international procedural capacity of individuals came to be exercised, with their direct access to international justice.

The work of contemporary international human rights tribunals, as well as international criminal tribunals, has given a new impetus to the struggle of the international community against impunity. The significance of the right of individual petition - a definitive conquest of the International Law of Human Rights ${ }^{66}$ - can only be properly assessed in historical perspective.In my aforementioned Separate Opinion in the recent ICJ Advisory Opinion on theRevision of a Judgment of the ILO Administrative Tribunal upon a Complaint Filed against IFAD(2012), I then turned my criticisms on what I perceive as the erosion of the strict inter-State outlook of adjudication by

65. This is exemplified by the system of the navigation of the river Rhine, by the Project of an International Prize Court (1907), by the Central American Court of Justice (19071917), as well as, in the era of the League of Nations, by the systems of minorities (including Upper Silesia) and of the territories under mandate, by the systems of petitions of the Islands Aaland and of the Saar and of Danzig, besides the practice of mixed arbitral tribunals and of mixed claims commissions, of the same epoch. For a study, cf., e.g.: A.A. Cançado Trindade, "Exhaustion of Local Remedies in International Law Experiments Granting Procedural Status to Individuals in the First Half of the Twentieth Century", 24 Netherlands International Law Review (1977) pp. 373-392; C.A. Norgaard, The Position of the Individual in International Law, Copenhagen, Munksgaard, 1962, pp. 109-128; M.St. Korowicz, Une expérience de Droit international - La protection des minorités de HauteSilésie, Paris, Pédone, 1946, pp. 81-174; among others.

${ }^{66}$. A.A. Cançado Trindade, El Derecho Internacional de los Derechos Humanos en el Siglo XXI, 1st. ed., Santiago, Editorial Jurídica de Chile, 2001, pp. 317-370. 
the of adjudication by the Hague Court (pars. 76-81 and 88-90). I pondered that the fact that the Advisory Committee of Jurists did not find, in 1920, that the time was ripe to grant access to the PCIJ to subjects of rights other than the States, such as the individuals, did not mean a definitive answer to the question at issue. The fact that the same position was maintained at the time of adoption in 1945 of the Statute of the ICJ did not mean a definitive answer to the question at issue.

The question of access of individuals to international justice, with procedural equality, continued to occupy the attention of legal doctrine ever since, throughout the decades. Individuals and groups of individuals began to have access to other international judicial instances (cf. supra), reserving the PCIJ and later the ICJ only for disputes between States. The dogmatic position taken originally in 1920, on the occasion of the preparation and adoption of its Statute, did not hinder the PCIJ to occupy itself promptly of cases pertaining to the treatment of minorities and inhabitants of cities or territories with a juridical statute of their own. In considerations developed in the examination of such matters, the PCIJ went well beyond the inter-State dimension, taking into account the position of individuals themselves (as in, e.g., inter alia, the Advisory Opinions on theJurisdiction of the Courts of Danzig, 1928, and on Minority Schools in Albania, 1935). Ever since, the artificiality of such dimension became noticeable and acknowledged, already at an early stage of the case-law of the PCIJ.

The exclusively inter-State character of the contentieux before the ICJ has not appeared satisfactory at all either. At least in some cases (from 1955 to 2013), pertaining to the condition of individuals, the presence of these latter (or of their legal representatives), in order to submit, themselves, their positions, would have enriched the proceedings and facilitated the work 
of the Court ${ }^{67}$. In those cases, one cannot fail to reckon that one of their predominant elements was precisely the concrete situation of the individuals directly affected, and not merely abstract issues of exclusive interest of the litigating States in their relations inter $s e^{68}$. Other illustrations can be found in the exercise of the advisory function of the ICJ, for example, in the ICJ two most recent Advisory Opinions, namely, the Advisory Opinion on the Declaration of Independence of Kosovo (2010), and the Advisory Opinion of the ICJ on the Revision of a Judgment of the ILO Administrative Tribunal upon a Complaint Filed against IFAD(2012).

In all these recent cases and Advisory Opinions, one cannot fail to recognize that a key element - at times the predominant one - has precisely been the concrete situation of human beings, and not mere abstract questions

67. One may recall, for example, the classical Nottebohm case concerning double nationality (Liechtenstein versus Guatemala, 1955), the case concerning the Application of the Convention of 1902 Governing the Guardianship of Infants, (The Netherlands versus Sweden, 1958), the cases of the Trial of Pakistani Prisoners of War (Pakistan versus India, 1973), of the Hostages (U.S. Diplomatic and Consular Staff) in Teheran case (United States versus Iran, 1980), of the East-Timor (Portugal versus Australia, 1995), the case of the Application of the Convention against Genocide (BosniaHerzegovina versus Yugoslavia, 1996), and the three successive cases concerning consular assistance - namely, the case Breard (Paraguay versus United States, 1998), the case LaGrand (Germany versus United States, 2001), the case Avena and Others (Mexico versus United States, 2004).

68. Moreover, one may further recall that, in the case of Armed Activities in the Territory of Congo (D.R. Congo versus Uganda, 2000) the ICJ was concerned with grave violations of human rights and of International Humanitarian Law; in the Land and Maritime Boundary between Cameroon and Nigeria (1996), it was likewise concerned with the victims of armed clashes.More recent examples wherein the Court's concerns have gone beyond the inter-State outlook include, inter alia, e.g., the case on Questions Relating to the Obligation to Prosecute or Extradite (Belgium versus Senegal, 2009) pertaining to the principle of universal jurisdiction under the U.N. Convention against Torture; the case of A.S. Diallo (Guinea versus D.R. Congo, 2010) on detention and expulsion of a foreigner; the case of the Application of the International Convention on the Elimination of All Forms of Racial Discrimination (Georgia versus Russian Federation, 2011), the case of the Temple of Preah Vihear (Cambodia versus Thailand, provisional measures of protection, 2011; and interpretation of judgment, 2014). 
of exclusive interest of the contending States in their relations inter se. The truth remains that the artificiality of the exclusively inter-State outlook of the procedures before the ICJ is clearly disclosed the very nature of some of the cases submitted to it. Such artificiality has been criticised, time and time again, in expert writing (cf. supra). Parallel to the construction of their international juridical personality, the access of individuals to contemporary international tribunals for theprotection of their rights reveals a renovation of international law - in the sense of its aforementioned humanization ${ }^{69}$, opening a great gap in the traditional doctrine of the reserved domain of States $^{70}$ (or compétence nationale exclusive), definitively overcome: the individual is erected as subject of international law, endowed with procedural capacity.

Before internationals tribunals, the human person encounters herself, to protect herself from the arbitrariness of the State, being protected by the rules of international law. This renovation of international law,proper of our time, corresponds to the recognition of the necessity that all States, in order to avoid newviolations of human rights, are to respond for the way they treat all human beings who are under their jurisdiction. Such renovation would simply not have been possible without the crystallization of the right of individual petition, amidst the recognition of the objective character of the obligations of protection and the acceptance of the collective guarantee of compliance with them: this is the real sense of the historical rescue of the individual as subject of the International Law of Human Rights (cf. supra).

69. Cf. A.A. Cançado Trindade, "El Nuevo Reglamento de la Corte Interamericana de Derechos Humanos (2000): La Emancipación del Ser Humano como Sujeto del Derecho Internacional de los Derechos Humanos", 30/31 Revista del Instituto Interamericano de Derechos Humanos (2001) pp. 45-71; A.A. Cançado Trindade, "Vers la consolidation de la capacitéjuridiqueinternationaledespétitionnaires...", op. cit. supran. (2), pp. 207-239.

${ }^{70}$. F.A. von der Heydte, "L'individu et les tribunaux internationaux", 107 Recueil des Cours de l'Académie de Droit International de La Haye (1962) pp. 332-333 and 329-330; and cf. A.A. Cançado Trindade, "The Domestic Jurisdiction of States in the Practice of the United Nations and Regional Organisations", 25 International and Comparative Law Quarterly (1976) pp. 715-765. 
In effect, it is of the essence of the international protection of human rights the counterposition between the complainant individuals and the respondent States in cases of alleged violations of the protected rights. The profound transformation of the international legal order, launched by the emergence of the International Law of Human Rights, has not taken place without difficulties, precisely for requiring a new mentality. It has furthermore undergone stages, some of which no longer sufficiently studied in our days, even in respect of the crystallization of the right of individual petition.Already in the beginnings of the exercise of this right it was stressed that, though motivated by the search for individual reparation, the right of petition contributes also to secure the respeet for obligations of an objective character which bind the States Parties.In various cases the exercise of the right of petition has gone further, ocasioning changes in the domestic legal order and in the practice of the public organs of the State. The significance of the right of individual petition can only be properly assessed in historical perspective.

Consideration of the right of individual petition as a method of international implementation of human rights necessarily takes into account the central aspect of the legitimatio ad causam of petitioners and the conditions of the exercise (and admissibility) of petitions (set forth in the distinct human rights instruments which foresee them $)^{71}$. In effect, of all the mechanisms of international protection of human rights, the right of individual petition is, effectively, the most dynamic one, attributing the iniciative of action to the individual himself (the ostensibly weaker party vis$\grave{a}$-vis the public power), distinctly from the exercise ex officio of other methods (such as those of reports and investigations) on the part of the organs

\footnotetext{
71. For an examination of the matter, cf. A.A. Cançado Trindade, Tratado de Direito Internacional dos Direitos Humanos, vol. I, op. cit. supra n. (20), pp. 68-87.
} 
of international supervision.It is the one which best reflects the specificity of the International Law of Human Rights, in comparison with other solutions proper of Public International Law.

Ultimately, all Law exists for the human being, and the law of nations is no exception to that, guaranteeing to the individual his rights and the respect for his personality, as well as the capacity to exercise his rights.Human rights do assert themselves against all forms or domination or arbitrary power.The human being emerges, at last, even in the most adverse conditions, as ultimate subject of Law, domestic as well as international. The case of the "Street Children" (case Villagrán Morales and Others versus Guatemala, 1999-2001), decided by the IACtHR, the first one of the kind in which the cause of the children abandoned in the streets was brought before an international human rights tribunal, and in which some of those marginalized and forgotten by this world succeeded to resort to an international tribunal to vindicate their rights as human beings, is truly paradigmatic, and gives a clear and unequivocal testimony that the International Law of Human Rights has nowadays achieved its maturity.

\section{The Historical Significance of the International Subjectivity of theIndividual}

The international juridical subjectivity of the human being, as foreseen by the so-called "founding fathers" of international law (the droit des gens), is nowadays a reality. At this beginning of the XXIst century, this highly significant conquest can be appreciated within the framework of the historical process of humanization of international law. On the basis of the right of individual petition is erected the juridical mechanism of emancipation of the human being vis-à-vis his own State for the protection of his rights in the ambit of the International Law of Human Rights, - an emancipation which constitutes, in our days, a true juridical revolution, 
which comes at last to give an ethical content to the norms of both domestic public law and international law.

The recognition of the direct access of the individuals to the international justice reveals, at this beginning of the XXIst century, the new primacy of the raison de l'humanité over the raison d'État, inspiring the historical process of humanization of international law ${ }^{72}$. The subjects of international law have, already for a long time, ceased to be reduced to territorial entities ${ }^{73}$. (...) It is perfectly possible to conceptualize as subject of international law, precisely, any person or entity, titulaire of rights and bearer of obligations, which emanate directly from norms of international law. It is the case of individuals, whose direct contacts - without intermediaries - with the international legal order are thus fostered and strengthened. This evolution is to be appreciated in a wider dimension. The expansion of international legal personality, nowadays encompassing that of individuals as active and passive subjects of international law, goes pari passu with the acknowledgment of accountability in international law.

The mechanical and thoughtless attachment to unfounded dogmas of the past becomes even more unsustainable, as the attention of contemporary international legal doctrine on the expansion of the international legal personality turns to the central position occupied today by the victimized individuals, giving unequivocal witness of the new jus gentium of our times $^{74}$, - as I sought to demonstrate in the General Course of Public

\footnotetext{
72 . A.A. Cançado Trindade, A Humanização do Direito Internacional, op. cit. supra n. (43), pp. 3-409.

73 . More than half a century ago, as acknowledged in the celebrated Advisory Opinion of the International Court of Justice on Reparations for Damages (1949), the advent of international organizations had put an end to the States' monopoly of the international legal personality and capacity, with all the juridical consequences which ensued therefrom, cf., for a general study on the matter, A.A. Cançado Trindade, Direito das Organizações Internacionais, $6^{\text {th }}$. ed., Belo Horizonte/Brazil, Edit. Del Rey, 2014, pp. 7-846.
}

74. A.A. Cançado Trindade, Évolution du Droit international au droit des gens - L'accès des particuliers à la justice internationale: le regard d'un juge, Paris, Pédone, 2008, pp. 81-184; 
International Law which I delivered in 2005 at the Hague Academy of International Law ${ }^{75}$. The State-centric world dreamed by E. de Vattel has ceased to exist a long time ago. The evolution of the law of nations, quite on the contrary, keeps on following its path in the XXIth century, with the access to international justice nowadays secured also to persons who found themselves in situations of the utmost vulnerability, if not entirely in defencelessness (cf. supra).

In reaction to the succession of acts of barbarism and of the recurring horrors throughout the XXth century and the beginning of the XXIst century, contemporary international legal doctrine has cared to open itself to the expansion of the international legal personality, and, accordingly, of the corresponding legal capacity, as well as, significantly, of the international responsibility. Contemporary jus gentium has been undergoing a historical process of humanization ${ }^{76}$, caring to instrumentalize itself against the manifest insufficiencies and the dangers of the State-centric outlook or of the surpassed strictly inter-State vision. To that effect, the International Law of Human Rights has much contributed, to the point of the phenomenon transcending the parameters of this latter, and permeating in our days the corpus juris of International Law as a whole.

Contemporary international case-law contains eloquent illustrations of the access of the human person to international justice in circumstances of considerable adversity, in cases pertaining to, e.g., undocumented migrants, children abandoned in the streets (cf. supra), members of peace communities

R. Portmann, Legal Personality in International Law, Cambridge, Cambridge University Press, 2010, pp. 126-128, 243, 271-277 and 283.

75. A.A. Cançado Trindade, "International Law for Humankind: Towards a New Jus Gentium - General Course on Public International Law - Part I', 316 RCADI (2005), chs. IXX, pp. 252-317.

76. A.A. Cançado Trindade,A Humanização do Direito Internacional, op. cit. supra n. (43), pp. 107-172. 
and others civilians in situations of armed conflict, internally displaced persons, individuals (including minors of age) under infra-human conditions of detention, members of dispossessed indigenous communities, among others. In such circumstances, the centrality of the suffering of the victims has become notorious with their access to justice at international level ${ }^{77}$.

In our days, effective use has been made of the international individual petition also in such situations ${ }^{78}$, - something which could hardly have been anticipated, in their days, by the draftsmen of international treaties and instruments of human rights, endowed with petitioning systems. On the other hand, such recent advances are not at all surprising, as the International Law of Human Rights is essentially victim-oriented. Such development is due, in my perception, to the awakening of the human conscience to the imperative of protection of the human person in these circumstances of extreme vulnerability. It is in such circumstances that such protection reaches its plenitude.

In effect, to this remarkable evolution I dedicate my recent book (of 2011), on the matter, published in Oxford ${ }^{79}$. I examine therein some cases adjudicated by the IACtHR in recent years, - a cycle of cases of massacres, with aggravating circumstances, wherein grave violations of human rights were planned and perpetrated in pursuance of State policies, forming a systematic practice of extermination of human beings. The adjudication of

77. Cf., on this particular point, A.A. Cançado Trindade, El Ejercicio de la Función Judicial Internacional - Memorias de la Corte Interamericana de Derechos Humanos, 3rd. ed., Belo Horizonte/Brazil, Edit. Del Rey, 2013, ch. XIX, pp. 163-169.

78 . Cf. A.A. Cançado Trindade, "The Right of Access to Justice in the Inter-American System of Human Rights Protection", 17 Italian Yearbook of International Law (2007) pp. 7-24; A.A. Cançado Trindade, "Die Entwicklung des interamerikanischen Systems zum Schutz der Menschenrechte", 70 Zeitschrift für ausländisches öffentliches Recht und Völkerrecht (2010) pp. 629-699.

79. A.A. Cançado Trindade, The Access of Individuals to International Justice, Oxford, Oxford University Press, 2011, pp. 1-236. 
those cases was launched by the historical Judgment of the IACtHR (of 14.03.2001) in the case of the massacre of Barrios Altos concerning Peru;to that Judgment followed the subsequent Judgments of the IACtHR in the cases of the massacres ${ }^{80}$. Thus, massacres and crimes of State (perpetrated by State agents as part of a State policy), which tended to fall into oblivion some decades ago, have more recently been brought to the cognizance of international human rights tribunals (such as the Inter-American and European Courts), in order to determine the responsibility of the State (under the respective regional Conventions) for grave violations of the protected human rights ${ }^{81}$.

Cases of the kind have also been lodged, to other effects, with other international tribunals, such as the international criminal ones (for the determination of individual international criminal responsibility), and the ICJ (in the framework of the inter-State contentieux). The current multiplicity of contemporary international tribunals (a reassuring phenomenon of our times) has by itself considerably increased the number of justiciables all over the world, fostering the access to international justice in our days, even in cases of the aforementioned gravity. New developments have in fact occurred lately in international legal procedures ${ }^{82}$, such as the ones

80. Namely, the cases of the massacres of Caracazo concerning Venezuela (reparations, of 29.08.2002), of Plan de Sánchez pertaining to Guatemala (of 29.04.2004), of the 19 Tradesmen versus Colombia (of 05.07.2004), of Mapiripán versus Colombia (of 17.09.2005), of the Moiwana Community versus Suriname (of 15.06.2005), of Pueblo Bello versus Colombia (of 31.01.2006), of Ituango versus Colombia (of 01.07.2006), of Montero Aranguren and Others (Detention Centre of Cátia) versus Venezuela (of 05.07.2006), of La Cantuta versus Peru (of 29.11.2006), and of the Prison of Castro Castro versus Peru (of 25.11.2006), as well as in the cases of assassinations planned at the highest level of the State power and executed by order of this latter (such as that of Myrna Mack Chang, Judgment of 25.11.2003).

81. For a recent study, cf. A.A. Cançado Trindade, State Responsibility in Cases of Massacres: Contemporary Advances in International Justice (Inaugural Address, 10.11.2011), Utrecht, Universiteit Utrecht, 2011, pp. 1-71.

82. Cf., in this respect, A.A. Cançado Trindade, "Reflexiones sobre los Tribunales Internacionales Contemporáneos y la Búsqueda de la Realización del Ideal de la Justicia 
pertaining to the determination of the aggravated international responsibility of the States concerned, and the identification of the víctims in distinct stages of those procedures.In reaction to grave violations of human rights, one may attest, in the international adjudication of such cases, the centrality and expansion of the notion of (direct) victim, and the relevance of their right to reparation for the damages suffered.It is highly significant that, in our days, surviving victims of massacres, and relatives of fatal victims, have had access to international justice.

States themselves today recognize and realize that they can no longer dispose, as they wish, of the human beings who happen to be under their respective jurisdictions ${ }^{83}$.Their power of action is not unlimited, ought to be guided by the faithful observance of certaing fundamental values ${ }^{84}$, and of the general princíples of law ${ }^{85}$.They are to respond for their eventual damages to human beings under their respective jurisdictions, and to provide the reparations due to them ${ }^{86}$. States cannot even shield themselves behind

Internacional", in Cursos de Derecho Internacional y Relaciones Internacionales de Vitoria-Gasteiz / Vitoria-Gasteizko Nazioarteko Zuzenbidearen eta Nazioarteko Harremanen Ikastaroak - Universidad del País Vasco (2010) pp. 17-95; A.A. Cançado Trindade, "Os Tribunais Internacionais Contemporâneos e a Busca da Realização do Ideal da Justiça Internacional”, 57 Revista da Faculdade de Direito da Universidade Federal de Minas Gerais (2010) pp. 37-67.

83. Cf. my Separate Opinion (paras. 1-231) in the Advisory Opinion of the ICJ on the Declaration of Independence of Kosovo (of 22.07.2010).

${ }^{84}$. Cf., e.g., S. Glaser, "La protection internationale des valeurs humaines", 60 Revue générale de Droit international public (1957) pp. 211-241.

85. Cf. my Dissenting Opinion (paras. 1-214) in the case of the Application of the International Convention on the Elimination of All Forms of Racial Discrimination (Georgia versus Russian Federation, Judgment of the ICJ of 01.04.2011); cf. also my Separate Opinion (paras. 1-184) in the recent case of Questions Relating to the Obligation to Prosecute or Extradite (Belgium versus Senegal, Judgment of the ICJ of 20.07.2012); and cf. my Separate Opinion (paras. 1-118) in the Advisory Opinion of the ICJ on the Revision of a Judgment of the ILO Administrative Tribunal upon a Complaint Filed against IFAD( of 01.02.2012).

${ }^{86}$. Cf. my Separate Opinion (paras. 1-101) in the case of A.S. Diallo (Guinea versus D.R. Congo, reparations, Judgment of the ICJ of 19.06.2012). 
the international criminal responsibility of the individuals who perpetrated international wrongs; the responsibility of the State always subsists. The responsibilities of ones and the others do not exclude each other, but rather complement each other. The new international legal order of our times has emerged from the human conscience, - the universal juridical conscience, as the ultimate material source of all Law.The expansion of the international legal personality has taken place to the benefit of all subjects of law, including the individuals as subjects of International Law.

\section{Epilogue}

The law of nations, the droit des gens, has much evolved, ultimately moved by the universal juridical conscience, which stands well above the will of the States. Those who serve States tend to think too highly of themselves, and to attribute a key role in this evolution to strategic international litigation.Hence their adherence to the unsatisfactory and dangerous inter-State framework, and to dogmas of the past, and their insistence on trying to prolong certain moments of legal history, without realizing that theirs is a static outlook of the law of nations. After all, their activity is one of means - to "win a case", - and not of ends. Those encapsulated in dogmatisms of the past tend to undermine advances achieved in the contemporary jus gentium, such as those pertaining to the consolidation of the international legal personality and capacity of individuals, which they label, at best, as "not perfect". They are longing for an international legal order which no longer exists.

Theirs is a static outlook, centred on States, unpersuasive for its arbitrary points of reference. Such outlook attempts in vain to minimize the remarkable evolution of international law itself, as illustrated, e.g., by the historical recovery of the human person as subjecto of the law of nations. 
That surpassed inter-State outlook discloses a far too limited view which nostalgically insists on what they regard as the "perfect" international legal personality of States, - a "perfectness" taken for granted, without demonstration. The heralds of that outlook tend to forget that States were created by human beings for their common good, and benefited from "personification" on the basis - ironically - of an analogy with that of human beings. It is about time that States do their part, to the benefit of human beings who created them for the common good.

The international legal order no longer fits itself into the straightjacket of the strict inter-State outlook, which led to so many abuses, and atrocities, in the recent past; it turns instead its attention, with the revival of jusnaturalism, to the condition of human beings, and the questions which affect the whole of humankind ${ }^{87}$. One recognizes today the need to consolidate the restitution to the uman person the central position - as subject of both domestic and international law-wherefrom she was unduly removed, with the disastrous consequences of sad memory. Therecognition of the centrality of the rights of the human person corresponds to a new ethos of our times. In this line of evolution also lies the current trend of "criminalization" of grave violations of the rights of the human person. At this early stage of the XXIst century, we witness the acceleration of the historical process of humanization of international law, ${ }^{88}$ - to which it is a privilege to be able to contribute, - which comes to occupy itself more directly with the realization of superior common goals.

${ }^{87}$. A. Truyol y Serra, La Sociedad Internacional, $9^{\text {th }}$. ed., Madrid, Alianza Editorial, 1998, pp. 97-98 and 167. On the conception of the Stateas promoter and guarantee of the common good,cf. Jacques Maritain, The Person and the Common Good, Notre Dame, University of Notre Dame Press, 1966 (reimpr. 1985), pp. 11-105.

${ }^{88}$ Cf. A.A. Cançado Trindade, A Humanização do Direito Internacional, op. cit. supra $\mathrm{n}$. (43), pp. 3-409. 
We stand before a humanized (or even truly humanist) international ordre public, wherein the public interest or the general interest coincides fully with that of the prevalence of human rights. That implies the recognition that human rights constitute themselves the basic foundation of the legal order. In the domain of the International Law of Human Rights, moved by considerations of international ordre public, we are before common and superior values, underlying it, and whuch appear truly fundamental and irreducible. We can here visualize a true droit au Droit, that is, the right to a legal order which effectively safeguards the rights inherent to the human person. Of their faithful safeguard will depend, to a large extent, the future evolution of International Law itself.

This is the path to follow, for us and the succeeding generations not to keep on living with the tragic contradictions which marked the XXth century. To pursue in this path, moreover, contributes ultimately to the international rule of law itself, to the realization of justice also at international level, thus fulfilling a long-standing aspiration of humankind. The international juridical subjectivity of the individuals is nowadays an irreversible reality, and the violation of their fundamental rights (emanated directly from the international legal order) entails juridical consequences. It gives expression to the new primacy of the raison d humanité over the raison $d$ État. Human conscience thereby attains in our days a stage of evolution which renders it possible to do justice at the international law in the safeguard of those entirely marginalized or socially excluded (cf. supra). The human person has emerged, at last, also in the most adverse conditions and even amidst defencelessness, as the ultimate subject of both domestic and international law, endowed with full juridico-procedural capacity.

Contemporary international law has been moved, in its advances, by the search for justice and for the prevalence of common superior values. It has purported to enable individuals to exercise their rights (by acknowledging their legitimatio ad causam) and peoples to live in peace with 
justice. The fact is that, nowadays, individuals, even in the most adverse circumstances, and in situations of defencelessness (as we have seen), have had access to international justice; this would have been simply unthinkable in the XIXth century, or even some decades ago. The international legal order nowadays promptly reacts to situations of manifest injustice. As jurists, we can give our modest contribution to the improvement of the human condition, beyond the confines of institutionalized "schools of thought", essentially as free thinkers, moved by our ideals, and remaining always attentive to fundamental human values, standing well above dogmas. Human conscience (the recta ratio), the universal juridical conscience, stands well above the will of States.

Last but not least, may I reiterate my gratitude to the authorities of the University Panteio of Athens for their kindness in convening this academic act, of great significance to me and which I shall never forget. Soon in my academic life I captured the longstanding legacy of Greek spirituality; this latter expressed, in a perennial and timeless way ${ }^{89}$, the compassion for the human condition, for human misery. The Greek tragedies of Aeschylus, Sophocles and Euripides remain as contemporary today as when they were first written and performed, so many centuries $\mathrm{ago}^{90}$. The message is clear: it is against the use and abuse of force, which

89. Cf., e.g., J. de Romilly, La Grèce antique contre la violence, Paris, Éd. de Fallois, 2000, pp. 7-214; J. Burckhardt, History of Greek Culture, Mineola/N.Y., Dover Publs., 2002, pp. 1-338; J.-P. Vernant, As Origens do Pensamento Grego, Rio de Janeiro, Difel/Ed. Bertrand, 2002, pp. 9-143; P. Lévêque, Le monde hellénistique, Paris, Libr. A. Colin,1969, pp. 3-264; B. Snell, A Cultura Grega e as Origens do PensamentoEuropeu [1955], São Paulo, Ed. Perspectiva, 2009 [reprint], pp. 1-319; J.N. Bremmer, The Early Greek Concept of the Soul, Princeton, Princeton University Press, 1993 [reprint], pp. 3135; R. Dodds, The Greeks and the Irrational, Berkeley, University of California Press, 1997 [reed.], pp. 1-311; H.D.F. Kitto, The Greeks, Middlesex, Penguin Books, 1964 [reprint], pp. 7-252; C.M. Bowra, The Greek Experience, N.Y., Mentor Books, 1959, pp. $13-215$.

${ }^{90}$. Cf., e.g., F. Nietzsche, The Birth of Tragedy [1872], Oxford, Oxford University Press, 2000, pp. 3-131; G. Steiner, The Death of Tragedy, London, Faber and Faber, 1961, pp. 
destroys not only the victims but likewise the perpetrators, everyone. It is a message illumitated by the imperatives of justice ${ }^{91}$. Justice is essential to human survival itself; we are privileged today to witness, and to contribute to, the emancipation of human beings vis-à-vis their own State, enabled as they now are to seek the realization of justice for themselves. Thank you very much for all your attention.

Athens, 01 July 2014.

A.A.C.T.

3-355; W. Kaufmann, Tragedy and Philosophy, Princeton, Princeton University Press, 1992 (reed.), pp. 1-379; J. de Romilly, Le temps dans la tragédie grecque, $2^{\text {nd }}$. ed., Paris, Libr. Philosophique J. Vrin, 2009, pp. 11-207; S. Goldhill, Sophoclesand the Language of Tragedy, Oxford, Oxford University Press, 2012, pp. 3-263; S. Goldhill, Reading Greek Tragedy, Cambridge,Cambridge University Press, 1999 (reed.), pp. 1-286.

91. Simone Weil, A Fonte Grega, Lisbon, Ed. Cotovia, 2006, pp. 16-17, 20, 24-25, 30, 37 39, 69-70, 106 and 127-128; Simone Weil, L'Tliade ou le poème de la force, Paris, Éd. Payot \& Rivages, 2014, pp. 54, 83, 86-87, 93, 101, 111-113, 119, 122-123, 142 and 152. 